\title{
Review Article \\ Gene Expression and Proteome Analysis as Sources of Biomarkers in Basal Cell Carcinoma
}

\author{
Mihai Lupu, ${ }^{1}$ Constantin Caruntu, ${ }^{2,3}$ Mihaela Adriana Ghita, ${ }^{4}$ Vlad Voiculescu, ${ }^{1}$ \\ Suzana Voiculescu, ${ }^{2}$ Adrian E. Rosca, ${ }^{2}$ Ana Caruntu, ${ }^{5}$ Liliana Moraru, ${ }^{5}$ Iris Maria Popa, \\ Bogdan Calenic, ${ }^{7}$ Maria Greabu, ${ }^{7}$ and Daniela Elena Costea ${ }^{8,9}$ \\ ${ }^{1}$ Department of Dermatology and Allergology, Elias Emergency University Hospital, 011461 Bucharest, Romania \\ ${ }^{2}$ Department of Physiology, "Carol Davila” University of Medicine and Pharmacy, 050474 Bucharest, Romania \\ ${ }^{3}$ Department of Dermatology, "Prof. N. C. Paulescu" National Institute of Diabetes, Nutrition and Metabolic Diseases, \\ 020475 Bucharest, Romania \\ ${ }^{4}$ Dermatology Research Laboratory, "Carol Davila” University of Medicine and Pharmacy, 050474 Bucharest, Romania \\ ${ }^{5}$ Department of Oral and Maxillofacial Surgery, "Carol Davila" Central Military Emergency Hospital, 010825 Bucharest, Romania \\ ${ }^{6}$ Department of Plastic and Reconstructive Surgery, "Bagdasar Arseni" Clinical Emergency Hospital, 041915 Bucharest, Romania \\ ${ }^{7}$ Department of Biochemistry, Faculty of Dental Medicine, University of Medicine and Pharmacy "Carol Davila", \\ 050474 Bucharest, Romania \\ ${ }^{8}$ Gade Laboratory for Pathology and Centre for Cancer Biomarkers (CCBio), Department of Clinical Medicine, \\ University of Bergen, 5021 Bergen, Norway \\ ${ }^{9}$ Department of Pathology, Haukeland University Hospital, 5021 Bergen, Norway
}

Correspondence should be addressed to Constantin Caruntu; costin.caruntu@gmail.com

Received 1 April 2016; Accepted 10 July 2016

Academic Editor: Caterina Longo

Copyright (C) 2016 Mihai Lupu et al. This is an open access article distributed under the Creative Commons Attribution License, which permits unrestricted use, distribution, and reproduction in any medium, provided the original work is properly cited.

Basal cell carcinoma (BCC) is the world's leading skin cancer in terms of frequency at the moment and its incidence continues to rise each year, leading to profound negative psychosocial and economic consequences. UV exposure is the most important environmental factor in the development of BCC in genetically predisposed individuals, this being reflected by the anatomical distribution of lesions mainly on sun-exposed skin areas. Early diagnosis and prompt management are of crucial importance in order to prevent local tissue destruction and subsequent disfigurement. Although various noninvasive or minimal invasive techniques have demonstrated their utility in increasing diagnostic accuracy of BCC and progress has been made in its treatment options, recurrent, aggressive, and metastatic variants of BCC still pose significant challenge for the healthcare system. Analysis of gene expression and proteomic profiling of tumor cells and of tumoral microenvironment in various tissues strongly suggests that certain molecules involved in skin cancer pathogenic pathways might represent novel predictive and prognostic biomarkers in BCC.

\section{Introduction}

Basal cell carcinoma (BCC) is the most common skin cancer worldwide and its incidence is still rising with almost $10 \%$ each year worldwide $[1,2]$, thus representing a growing public health problem associated with negative psychosocial and economic consequences $[3,4]$.

These tumors that develop de novo have relatively uniform histology, and while not lethal they are locally invasive causing disfigurement and increasing morbidity due to frequent facial localization. Early diagnosis and prompt management are of crucial importance in order to prevent local tissue destruction or the occurrence of advanced disease. Although histopathological examination is considered the gold standard of diagnosis for BCC and other skin tumors, noninvasive and minimal invasive diagnostic tools have gained increased attention, as they do not imply performing a skin biopsy [5]. Among these novel optical imaging 
techniques, dermoscopy and reflectance confocal microscopy allow a rapid, in vivo, noninvasive micromorphological evaluation of skin tumors and combining these techniques can increase the diagnostic accuracy in different subtypes of BCC [6-8].

Known risk factors for developing BCCs include Fitzpatrick type I phototype, freckling and sunburns in childhood, family history of skin cancer, iatrogenic immunosuppression, and internal or external exposure to carcinogenic chemicals, especially arsenic [9] and high cumulative exposure to UV light [10]. Among these, UV radiation is considered by most as the main carcinogen [11] and around $80 \%$ of BCCs occur on sun-exposed areas, mostly the head and neck.

Regional anatomical differences, such as type and density of hair follicles, could explain why the back of the hands, despite extensive sun exposure, is a rare location for these tumors.

Carcinogenic processes $[12,13]$ are likely the result of multifarious interactions between host genome and environmental factors. Because of its morphological similarities to the undifferentiated epidermal basal cells, BCC provides an excellent model for identifying differentially expressed genes when compared to normal cells [14]. Genomic and proteomic techniques have both the potential to deliver new biomarkers [15]. However, gene transcript levels do not always correlate with protein expression due to transcriptional/translational control and high-throughput proteomic technologies are preferred for the measurement of proteins associated with pathological states [16]. Moreover, in early stages of the neoplastic process, individual proteomic platforms or platform combinations are used to characterize the great number of intact and cleaved proteins that can separate patients from healthy subjects [17]. Further, novel soluble and/or tissuespecific biomarkers can be developed for diagnosis, prognosis, and therapy monitoring in various malignancies [18-20], including BCC and other nonmelanoma skin cancers [21].

\section{Sporadic Basal Cell Carcinoma}

Multiple signaling pathways are altered in carcinogenesis [22], and changes that lead to sonic hedgehog $(\mathrm{SHH})$ patched 1 signaling pathway dysregulation have been recognized as essential events for sporadic BCC development [23] (see Figure 1). Gene expression alteration is an important event in tumor cells and mutations in the patched 1 gene are frequently present in BCCs [14].

Glil (glioma 1) transcription factor is an important downstream effector in the $\mathrm{SHH}$ pathway and has regularly been found upregulated in basal cell carcinomas [23].

GlI2 (glioma 2) is another key protein in BCC carcinogenesis and is also required for normal hair follicle development in the embryonic stages [24]. It has been proved that overexpression of GII2 in the basal keratinocytes of transgenic mice leads to the development of multiple BCC-like tumors [25]. In addition, GLI2 has been regularly found upregulated in cancers presenting complex genomic alterations [26].

FOXM1, a Forkhead box protein, is a downstream molecule of SHH/Glil that has been found to be overexpressed in BCC and because of its role in cell proliferation it is thought to be one of the causes for aberrant $\mathrm{SHH}$ signaling in BCC tumorigenesis [14].

Lam et al. found another Forkhead box protein, FOXO3A, to be overexpressed in BCCs. FOXO3A, a transcription factor known for its involvement in cell-cycle arrest mediation, apoptosis, and DNA repair [27], also plays a key role in oxidative stress protection through upregulation of several antioxidants, such as SOD2 and catalase.

In a 2008 study Asplund et al. compared gene expressions of normal epidermal basal cells to those of BCC cells. The results revealed 201 upregulated and 160 downregulated genes in BCC cells compared to normal basal cells. Among them, they identified differentially expressed genes implicated in cell differentiation (aquaporin 3 and envoplakin), adhesion (claudin-1 and CD44), communication (desmoglein 2), and immune response (CD40 and MHC class II proteins). Consistent differences in immunoreactivity between tumor and control cells were found in half of gene products: claudin-1, cystatin A, CD44, calgranulin A (S100A8), prostaglandin-endoperoxide synthase 1 (COX-1), junctional adhesion molecule 3 (JAM3), envoplakin, and cmyc. Several members of the Wnt pathway were also found to be upregulated: Wnt receptor frizzled $8, \beta$-catenin, Lef1 , and basonuclin-2. Desmoglein 2, a desmosomal cadherin strongly expressed in the bulge area and basal layer of the outer root sheath [28] and weakly in interfollicular epidermis [29], was also found to be overexpressed in BCC tumor cells. Several proteins were downregulated, namely, CD40, MHC class II molecules, Fc fragment of IgG binding protein, and immunoglobulin superfamily member 1 [14].

Zali [30] found that $\mathrm{C} 3 \mathrm{~b}$, which has important roles in opsonisation and activation of the alternate pathway [31], had elevated expression levels in BCC cells when compared to controls, while transthyretin and ceruloplasmin had lower expressions. These results are supported by a previous study that found ceruloplasmin significantly decreased in BCC patients compared to healthy controls [32], probably owing to prolonged exposure to ultraviolet radiation. As no expression for aldolase C, FGG (Fibrinogen gamma chain), Prx-cis (periaxin), prothrombin, VDAC (voltage-dependent anion channels), and LRG (leucine-rich alpha-glycoprotein) was found in tumor cells, they were considered negative markers for BCC. In addition, the author underlines the necessity for further studies of these biomarkers in mucous secretions and blood [30].

\section{Recurrent and Subsequent Basal Cell Carcinomas}

Although various risk factors for increased incidence of recurrent BCC exist [33], none of them should be considered individually. Clinical risk factors for BCC recurrence include male sex, lesion topography (centrofacial region involving the inner canthus, nostrils, and periauricular area), tumor size (recurrence rate increases by $7 \%$ for each millimeter of increase in tumor diameter), photosensitivity, and over 60 years of age at first presentation $[34,35]$. Aggressive-growth morphologic variants such as sclerosing, mixed (nodular and 


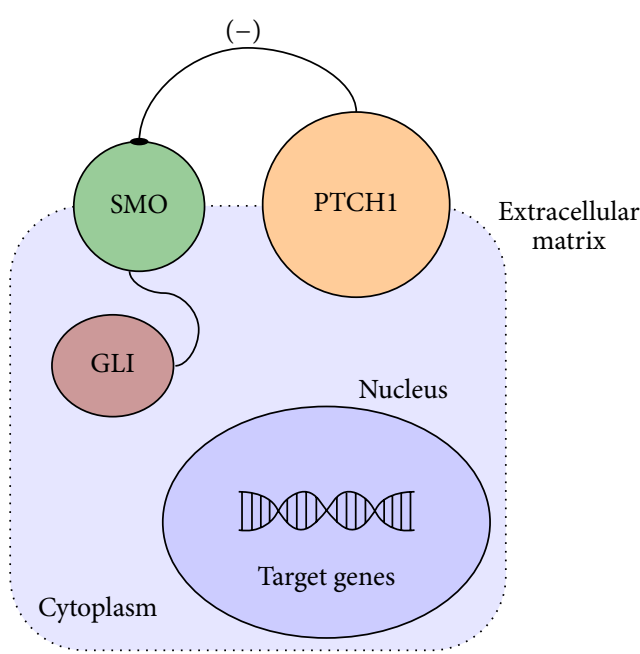

(a) Inactive

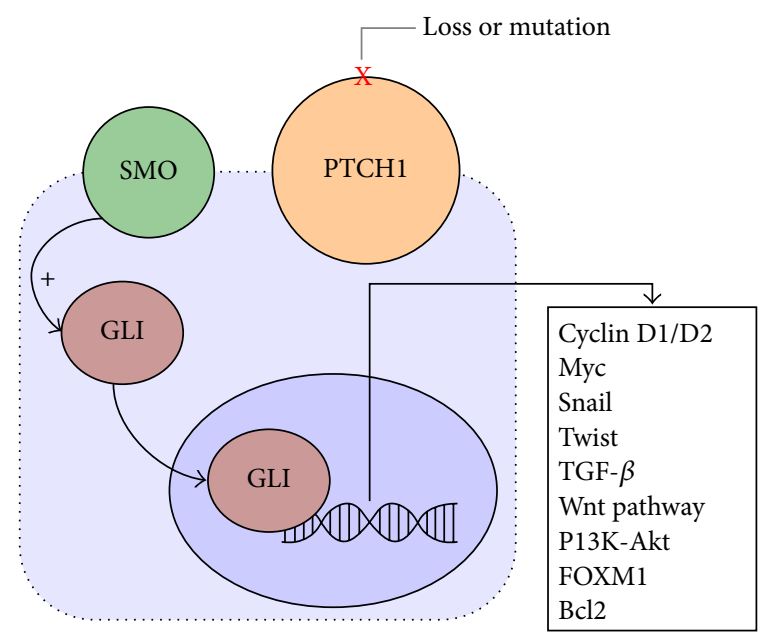

(c) Oncogenic signaling in BCC, ligand independent, PTCH1 loss or mutation

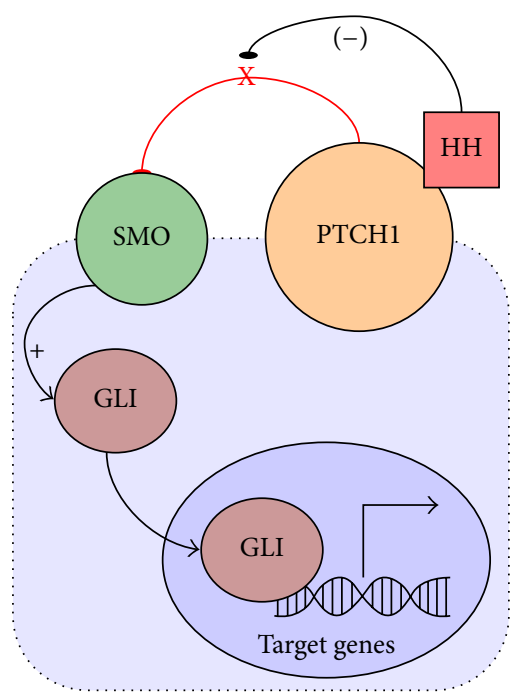

(b) Physiologically activated, ligand dependent, reversible

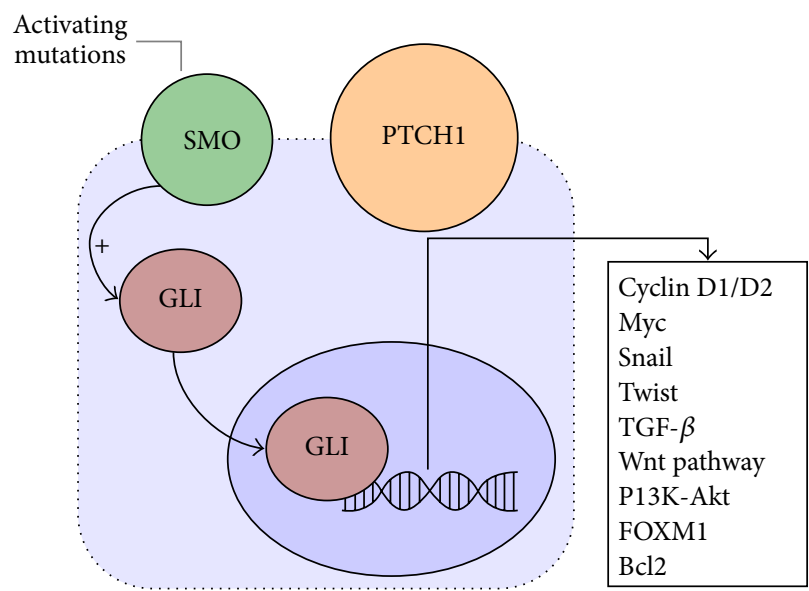

(d) Hedgehog pathway activation through SMO activating mutations

FIGURE 1: Sonic hedgehog (SHH) signaling pathway. (a) In the absence of the HH ligand, PTCH1 represses signaling through SMO causing GLI to remain inactive in the cytoplasm. (b) During physiological activation of the pathway the HH ligand binds to PTCH1, ending SMO suppression and causing activation and nucleus translocation of GLI thus influencing target genes expression. (c) Pathological activation of the SHH pathway through loss or mutational inactivation of PTCH1, suspending SMO inhibition in Gorlin-Goltz and sporadic BCCs (twist, FOXM1, Wnt pathway molecules, and others are viable biomarkers in BCC proteomic studies). (d) SMO activating mutations, found in sporadic BCCs, showing similar effects on the SHH pathway. HH: sonic hedgehog ligand; PTCH: protein patched hedgehog receptor, SMO: smoothened receptor, GLI: GLI factor, and Wnt: wingless signaling pathway.

sclerosing), and less commonly superficial multifocal type are considered by some $[36,37]$ to be histological risk factors.

A proteomic approach of potential predictive markers for BCC recurrence employed the study of cyclooxygenase-2, ezrin, and matrix metalloproteinase-9 [33].

One study found that overexpression of COX-2 plays a significant role in carcinogenesis through several mechanisms such as enhancement of cellular proliferation, promotion of angiogenesis, inhibition of apoptosis, stimulation of invasion, and suppression of immune responses [38]. The promotion of tumor cell proliferation appears to result from cooperation between COX-2 and various cellular signaling pathways [39, 40]. Moreover, a connection between COX2 overexpression and increased levels of vascular endothelial growth factor-A (VEGF-A), CD31 positive vessels, and regulators of apoptosis $\mathrm{Mcl}-1$ and $\mathrm{Bcl} 2$ has been implied by previous studies [41]. El-Khalawany and Abou-Bakr found that $90.9 \%$ of recurrent BCCs (rBCC) expressed COX-2 compared to only $59.1 \%$ of nonrecurrent BCCs (nrBCC) [33]. Conversly, it was shown that COX-2 inhibition can mitigate tumor growth, decrease the expression cell proliferation markers, and promote cancerous cell apoptosis $[39,40]$. 
Ezrin is a cytoplasmic peripheral membrane protein belonging to the ERM (ezrin, Radixin, and Moesin) protein family and acts as an intermediate between the plasma membrane and the actin cytoskeleton having a significant role in cell surface structure adhesion, migration, organization, tumor growth regulation and progression, and metastatic spread of numerous cancers [42, 43]. Higher frequencies for ezrin immunopositivity have been observed in rBCCs than in nrBCCs [33]. While Bagheri and collaborators found ezrin expression in $93 \%$ of the tested BCCs, no significant difference regarding ezrin expression was observed between different BCC subtypes. The authors did note a difference between the intensity of staining between BCC subtypes, with morpheaform BCC having a significantly higher intensity of staining compared to the nodular variant [44]. Some studies reported a higher ezrin expression in SCC compared with less aggressive tumors such as solar keratosis, keratoacanthoma, or Bowen's disease [42]. Moreover, in primary cutaneous melanomas ezrin expression was found to be associated with depth of invasion, progression, and metastasis [45]. These results are supported by a previous study [46] that found $92 \%$ positivity for ezrin expression in 25 BCCs. The authors, however, did not find any difference regarding positivity or staining pattern between BCCs and SCCs. They hypothesized that the intensity rather than the pattern of ezrin expression had a more probable impact on the tumor behavior but admitted that larger studies are needed for clarification.

Matrix metallopeptidase 9 (MMP-9), an enzyme also known as $92 \mathrm{kDa}$ type IV collagenase, is a matrixin encoded by MMP-9 gene. Along with other molecules, it plays an important role in neutrophil migration across the basement membrane [47], angiogenesis, and neovascularization in tumoral tissues through recruitment of endothelial stem cells [48] and wound repair by stimulation collagen contraction [49]. MMP-9 was detected by ISH (in situ hybridization) in the stromal fibroblasts adjacent to tumor invasion sites in infiltrating basal cell and squamous cell carcinomas and in the eosinophils infiltrating the dermis in response to invasive BCC $[50,51]$ and another study found an increased expression of MMP-9 and MMP-2 in SCC versus BCC [52]. Dumas et al. considered the reduced expression of collagen IV accompanied by the increased expression of MMP-9 and MMP-2 could explain the increased aggressive behavior of SCC over BCC [52]. One study [33] did not find any statistically significant difference between MMP-9 expressions in recurrent versus nonrecurrent BCCs.

In a prospective study, Glaser et al. [53] measured the levels of mRNA for CD3e (a T-cell surface marker), CD25 (alpha chain of IL-2 receptor expressed on activated T-cells and B-cells), CD68 (marker for monocytes/macrophages), the cell surface glycoprotein ICAM-1 (intercellular adhesion molecule-1), and the cytokines interferon-gamma (IFN- $\gamma$ ) and IL-10 in BCC tumors from 138 patients. The median follow-up in this study was 26.6 months. It was revealed that subjects with initially low CD3e, CD25, CD68, and ICAM-1 mRNA levels had a significantly shorter tumor-free period ( $p=0.03, p=0.02, p=0.003$, and $p=0.08$, resp.). It was also observed that nodular morphologies had lower gene expression levels compared to superficial or mixed tumors.
The authors could not link IFN- $\gamma$ mRNA levels to the risk of subsequent tumors [53]. This information shows that immune cell related gene expression in an initial BCC tumor could be used to predict subsequent BCC development. These results have been confirmed by other studies [54] which found elevated mRNA levels of IFN- $\gamma$, IL-2, and CD3e in regressing BCCs.

Hunt et al. conducted a study of primary BCCs with and without histological evidence of regression, proposing that some tumors induced immune responses capable of tumor disruption. They reported a significant increase in CD3 in tumors that present active regression compared to those showing no regression. They also found that expression of CD25 was greater in actively regressing BCCs compared to tumors that had no current or past regression [55].

These findings reinforce the importance of inflammatory and immune cells in tumor progression mediation.

\section{Aggressive, Metastatic, and Giant Basal Cell Carcinoma}

Numerous studies have employed proteomics in their attempt to characterize aggressive BCC and distinguish it from nonaggressive variants.

Ansarin et al. [56] found that elevated p53 protein expression could be considered a predictor of BCC aggressive behavior. However, another study [57] reported that even though differences in p53 gene mutation frequency, types of mutations, and hot spots between aggressive and nonaggressive BCC exist, they do not clearly predict tumor behavior. Yu et al. [58], in a 2008 gene expression study, found that nodular and superficial BCCs demonstrate similar transcriptional profiles, but different from the morpheaform subtype, which shows a more diverse gene expression pattern, reflecting its invasive nature. However, Howell et al. [59] could not distinguish nodular from sclerosing BCC subtypes by their gene expression patterns.

As a common trait to all epithelial-derived tumors, BCC can express transcription factors like Snail and Twist 1 or mesenchymal markers like the cell adhesion molecule $\mathrm{N}$ cadherin.

The basic helix-loop-helix (bHLH) transcription factor Twist 1 was initially identified in an experimental tumor model as a major regulator of epithelial to mesenchymal transition (EMT) [60]. It was also found to be significantly upregulated in patients with metastatic breast cancer when compared to early disease stages [61].

Epithelial to mesenchymal transition (EMT) is a complex process by which cells lose their epithelial traits and gain a mesenchymal-like phenotype. Numerous factors, such as transforming growth factor beta (TGF- $\beta$ ), epidermal growth factor (EGF), and Wnt-b signaling, have been described to promote the expression of transcription factors Twist 1 and Snail in epithelial cells, resulting in decreased expression of E-cadherin, upregulation of N-cadherin, vimentin, and fibronectin and the acquisition of morphological and functional characteristics of mesenchymal tissue cells [62].

In 2012, Majima et al. [63] present a case of morphoeic and multiple organ metastatic BCC exhibiting induction of Twist 
1 and epithelial to mesenchymal conversion of cadherins in a 51-year-old Japanese male. Twist 1 expression was analyzed by immunohistochemistry on formalin-fixed paraffinembedded sections of the tumor with representative sections from nodular BCCs serving as controls. Cells at the invasive front of the primary tumor proved to be positive for Twist 1, whilst cells from the tumor center were negative for this marker. Control cells from nonmetastatic nodular BCCs did not show nuclear Twist 1 expression. Additionally, cadherins (E-cadherin and N-cadherin) were assessed by double immunofluorescence stains that showed strongly expressed E-cadherin in nonmetastatic nodular BCC and very low expression of this epithelial marker in cells from the metastatic BCC. By comparison, while no N-cadherin expression could be detected in the control tumors, cells from the metastatic BCC were found to express N-cadherin at the invasive front. Cells from a metastasis showed high expression levels of Twist 1 and $\mathrm{N}$-cadherin and notably decreased expression of E-cadherin. It has been previously shown that E-cadherin, a calcium-dependent cell adhesion molecule, plays a crucial role in tumor invasion suppression, and its loss of function is associated with increased tumor aggressiveness [64]. Pizarro reported reduced E-cadherin expression in infiltrative BCCs [65] that have also been previously shown to produce MMP-7 [66]. The authors consider Twist 1 a viable biomarker for either highly invasive or metastatic BCC [63]. Sasaki and collaborators found that in ESCC (esophageal squamous cell carcinoma) Twist or Ecadherin expression was correlated with tumor attributes, such as tumor stage, depth of invasion, lymphatic invasion, and regional and distant node metastasis, making their evaluation useful for determining prognosis [67].

Maspin, a protease inhibitor and a member of the serpin family, is the product of a tumor suppressor gene with an active role in apoptosis and inhibition of tumor invasion, metastasis, and angiogenesis [68]. Whereas Bagheri et al. [44] report expression of maspin in $74.4 \%$ of their samples of BCCs, they could not find a significant difference between BCC subtypes concerning maspin expression and intensity of staining. These results are in accordance with another study [69] in which maspin expression in BCC was found to be $87.5 \%$. Although biased by the inclusion of 3 metatypical BCCs, Abdou et el. report a maspin expression in BCC of only $48 \%[70]$.

Rates for metastatic BCC are around the 0.55\% mark, making it a very rare occurrence [71]. Defined by the AJCC (American Joint Committee on Cancer) as a tumor larger than $5 \mathrm{~cm}$ in diameter, giant BCC is also a very rare variant [72].

Alpha-smooth muscle ( $\alpha$-SMA) actin, an isoform typical of smooth muscle cells (SMC) encoded by the ACTA2 gene on chromosome 10q22-q24, is present in the skin in the arrector pili muscles, fibroblasts surrounding anagen hair follicles, myoepithelial cells of eccrine glands, perivascular pericytes, and vascular smooth muscle [73]. In several studies [74, 75] the expression of $\alpha$-SMA was significantly higher in the stroma of aggressive BCCs when compared to nonaggressive BCCs. Adegboyega et al. implied that stromal expression of $\alpha$-SMA was in fact restricted to aggressive tumors at the same time being highly predictive of aggressive behavior [74].
Recently, Motegi et al. studied the expression of Twist 1 and $\alpha$-SMA in the stromal cells of a metastatic giant basal cell carcinoma. They concluded that although Twist 1 induced EMT of tumor cells might have been linked to distant organ metastases in their case, the presence of $\alpha$ SMA in myofibroblasts surrounding BCC tumoral cell nests could certainly represent one of the trademarks of BCC aggressiveness [76].

Oh et al. suggested that membrane type- 1 matrix metalloproteinase (MT1-MMP) and $\beta$-catenin could be considered biomarkers for high-risk BCC due to their important role in locally invasive and destructive growth tumor behaviour [77]. El-Bahrawy et al. noted that $\beta$-catenin is found mainly in the membrane of tumor cells of high-risk BCC and suggested that a molecular mechanism, other than the aberrant Ecadherin/catenin complex, is involved in these high-risk subtypes of BCC [78].

ER homeostasis in tumoral cells is dysregulated by either physiological or pathological stimuli, such as oxidative stress, DNA-damage, nutrient deficiency, calcium-depletion, certain growth factors, and oncogenic factors. Under such conditions, unfolded and misfolded proteins accumulate, leading to ER stress and the activation of ER-specific signaling pathways [79].

Endoplasmic reticulum protein 29 (ERp29) is a chaperone protein found in the lumen of the endoplasmic reticulum (ER) [80]. It is thought to play a role in protein processing and transport in the early secretory pathway. Erp29 is expressed at varying levels practically in every tissue, yet its precise role in the pathogenesis of neoplasia remains unknown [81].

One study pointed out the tumor suppressive role of ERp29 demonstrated by inhibition of tumor formation in mice xenografts. The authors also suggested that overexpression of ERp29 could indirectly result in activation of genes with tumor suppressive functions, like E-cadherin and spleen tyrosine kinase [82].

Cheretis et al. studied the implication of ERp29 in the pathogenesis of cutaneous BCC. The results revealed that $37.5 \%$ of all analyzed tumors expressed ERp29. Infiltrating carcinomas displayed more intense immunoreactivity compared to superficial variants which displayed less intense anti-ERp29 staining [81]. According to this study ERp29 is expressed in a subset of BCC in which the infiltrating morphologies exhibit the highest incidence of immunopositivity.

Following cell metastasis, disseminated mesenchymal cancer cells can be reversibly converted to an epithelial cell state by mesenchymal-epithelial transition (MET) [83]. Considering that ERp29 can drive MET in mesenchymal breast cancer cells [82], ERp29 may play an important role in promoting distant metastasis during disease progression in basal cell carcinoma.

\section{Nevoid Basal Cell Carcinoma Syndrome (Gorlin-Goltz Syndrome)}

The molecular mechanisms underlying the pathogenesis of multiple BCCs in Gorlin-Goltz syndrome (GS) differ considerably from those of sporadic BCC development. Patients 
suffering from GS develop multiple BCCs at young ages and tumors are more often localized on non-UV-exposed skin [84]. Genetic anomalies of the PTCH1 gene in GS include nonsense, frameshift, in-frame, splice-site, interstitial, and missense mutations [85]. Immunohistochemical analysis with nonmutated site-targeting anti-PTCH1 antibody cannot differentiate GS associated from sporadic forms of BCC, because both show comparable patterns and intensity of staining [86].

A comparison of whole genome expression [87] between GS and healthy controls revealed a genomic signature which included several genes with known associations with tumor growth and invasiveness. The authors also report that the genotype of PTCH1+ fibroblasts from tumor-free skin of NBCCS patients was similar to that of BCC associated fibroblasts to the extent that NBCCS fibroblasts overexpressed mRNAs encoding MMP-1, MMP-3, and tenascin C, proproliferative factors such as fibroblast growth factor 7 (FGF-7) and stromal cell-derived factor 1 alpha. In addition, there was strong MMP-1 overexpression in PTCH1+ fibroblasts obtained from NBCCS patient compared to healthy donors [87].

Ponti et al. [88] analyzed profiles of fibroblast conditioned culture media of PTCH1+ and compared them to nonmutated fibroblasts. Statistically significant differences between two different types (missense versus nonsense) of PTCH1 mutations in the profiles of fibroblasts from conditioned media were revealed. Results confirmed previously documented [87] MMP-1 overexpression in PTCH1 mutated fibroblasts, thus confirming the relationship between PTCH1 mutation and MMP-1-related neoplastic transformation of epithelial cells.

Adding to these findings, matrix metalloproteinase-1 (MMP-1) was identified by Weiss et al. to be a downstream target of Twist 1. According to their study, MMP-1 may also enhance cellular motility and invasion of BCC by disrupting the basement membrane and degrading the stromal matrix [89].

MMP-1 can thus be considered a novel marker for target therapy in the context of NBCSS. Future studies of this particular proteomic signature could prove useful for the clinical, therapeutic, and prognostic evaluation of these patients.

\section{Conclusions}

In summary, it is apparent that gene expression alteration induced by different pathways in tumor cells due to the variation in the expression of other factors is an essential event in BCC carcinogenesis. Analyzing gene expression and proteomic profiles of tumor cells and its microenvironment in different tissue or fluid biological samples indicates new candidate molecules involved in skin cancer pathogenic pathways that might represent future predictive and prognostic biomarkers in BCC and other skin cancers.

\section{Competing Interests}

The authors declare that there is no conflict of interests regarding the publication of this paper.

\section{Authors' Contributions}

All authors have equally contributed to the conception and preparation of the paper.

\section{Acknowledgments}

This paper is partly supported by Grant PNII-PT-PCCA2013-4-1386 (Project 185/2014) financed by Executive Agency for Higher Education, Research, Development and Innovation and by Young Researchers Grant 33891/2014 financed by Carol Davila University of Medicine and Pharmacy, Bucharest. Bogdan Calenic acknowledges that this work was supported by a grant of the Romanian National Authority for Scientific Research and Innovation, CNCS-UEFISCDI, Project no. PN-II-RU-TE-2014-4-1879.

\section{References}

[1] A. Lomas, J. Leonardi-Bee, and F. Bath-Hextall, "A systematic review of worldwide incidence of nonmelanoma skin cancer," British Journal of Dermatology, vol. 166, no. 5, pp. 1069-1080, 2012.

[2] V. Madan, J. T. Lear, and R.-M. Szeimies, "Non-melanoma skin cancer," The Lancet, vol. 375, no. 9715, pp. 673-685, 2010.

[3] N. R. Telfer, G. B. Colver, and C. A. Morton, "Guidelines for the management of basal cell carcinoma," British Journal of Dermatology, vol. 159, no. 1, pp. 35-48, 2008.

[4] I. Hoorens, K. Vossaert, K. Ongenae, and L. Brochez, "Is early detection of basal cell carcinoma worthwhile? Systematic review based on the WHO criteria for screening," British Journal of Dermatology, vol. 174, no. 6, pp. 1258-1265, 2016.

[5] O. Warszawik-Hendzel, M. Olszewska, M. Maj, A. Rakowska, J. Czuwara, and L. Rudnicka, "Non-invasive diagnostic techniques in the diagnosis of squamous cell carcinoma," Journal of Dermatological Case Reports, vol. 9, no. 4, pp. 89-97, 2015.

[6] C. Longo, A. Lallas, A. Kyrgidis et al., "Classifying distinct basal cell carcinoma subtype by means of dermatoscopy and reflectance confocal microscopy," Journal of the American Academy of Dermatology, vol. 71, no. 4, pp. 716-724.el, 2014.

[7] C. Căruntu, D. Boda, D. E. Guţu, and A. Căruntu, "In vivo reflectance confocal microscopy of basal cell carcinoma with cystic degeneration," Romanian Journal of Morphology and Embryology, vol. 55, no. 4, pp. 1437-1441, 2014.

[8] M. A. Ghita, C. Caruntu, A. E. Rosca et al., "Reflectance confocal microscopy and dermoscopy for in vivo, non-invasive skin imaging of superficial basal cell carcinoma," Oncology Letters, vol. 11, no. 5, pp. 3019-3024, 2016.

[9] C. S. M. Wong, R. C. Strange, and J. T. Lear, "Basal cell carcinoma," British Medical Journal, vol. 327, no. 7418, pp. 794798, 2003.

[10] M. D. Abeloff, J. O. Armitage, and J. E. Niederhuber, Clinical Oncology, Elsevier Churchill Livingstone, 2004.

[11] R. Corona, E. Dogliotti, M. D’Errico et al., "Risk factors for basal cell carcinoma in a Mediterranean population: role of recreational sun exposure early in life," Archives of Dermatology, vol. 137, no. 9, pp. 1162-1168, 2001.

[12] A. Kricker, B. K. Armstrong, D. R. English, and P. J. Heenan, "A dose-response curve for sun exposure and basal cell carcinoma," International Journal of Cancer, vol. 60, no. 4, pp. 482-488, 1995. 
[13] M. Neagu, C. Caruntu, C. Constantin et al., "Chemically induced skin carcinogenesis: updates in experimental models," Oncology Reports, vol. 35, no. 5, pp. 2516-2528, 2016.

[14] A. Asplund, M. Gry Björklund, C. Sundquist et al., "Expression profiling of microdissected cell populations selected from basal cells in normal epidermis and basal cell carcinoma," British Journal of Dermatology, vol. 158, no. 3, pp. 527-538, 2008.

[15] C. Caruntu, D. Boda, G. Dumitrascu, C. Constantin, and M. Neagu, "Proteomics focusing on immune markers in psoriatic arthritis," Biomarkers in Medicine, vol. 9, no. 6, pp. 513-528, 2015.

[16] O. J. Freeman, R. D. Unwin, A. W. Dowsey et al., "Metabolic dysfunction is restricted to the sciatic nerve in experimental diabetic neuropathy," Diabetes, vol. 65, no. 1, pp. 228-238, 2016.

[17] M. Neagu, C. Constantin, C. Tanase, and D. Boda, "Patented biomarker panels in early detection of cancer," Recent Patents on Biomarkers, vol. 1, no. 1, pp. 10-24, 2011.

[18] C. P. Tanase, M. Neagu, R. Albulescu, and M. E. Hinescu, "Advances in pancreatic cancer detection," Advances in Clinical Chemistry, vol. 51, pp. 145-180, 2010.

[19] T. Janvilisri, K. Leelawat, S. Roytrakul, A. Paemanee, and R. Tohtong, "Novel serum biomarkers to differentiate cholangiocarcinoma from benign biliary tract diseases using a proteomic approach," Disease Markers, vol. 2015, Article ID 105358, 11 pages, 2015.

[20] R. M. Sallam, "Proteomics in cancer biomarkers discovery: challenges and applications," Disease Markers, vol. 2015, Article ID 321370, 12 pages, 2015.

[21] A. Bulman, M. Neagu, and C. Constantin, "Immunomics in skin cancer-improvement in diagnosis, prognosis and therapy monitoring," Current Proteomics, vol. 10, no. 3, pp. 202-217, 2013.

[22] B. Calenic, M. Greabu, C. Caruntu, C. Tanase, and M. Battino, "Oral keratinocyte stem/progenitor cells: specific markers, molecular signaling pathways and potential uses," Periodontology 2000, vol. 69, no. 1, pp. 68-82, 2015.

[23] M. Nilsson, A. B. Undèn, D. Krause et al., "Induction of basal cell carcinomas and trichoepitheliomas in mice overexpressing GLI-1," Proceedings of the National Academy of Sciences of the United States of America, vol. 97, no. 7, pp. 3438-3443, 2000.

[24] P. Mill, R. Mo, H. Fu et al., "Sonic hedgehog-dependent activation of Gli2 is essential for embryonic hair follicle development," Genes and Development, vol. 17, no. 2, pp. 282-294, 2003.

[25] M. Grachtchouk, R. Mo, S. Yu et al., "Basal cell carcinomas in mice overexpressing Gli2 in skin," Nature Genetics, vol. 24, no. 3, pp. 216-217, 2000.

[26] A. Ruiz i Altaba, P. Sánchez, and N. Dahmane, "Gli and hedgehog in cancer: tumours, embryos and stem cells," Nature Reviews Cancer, vol. 2, no. 5, pp. 361-372, 2002.

[27] E. W.-F. Lam, R. E. Francis, and M. Petkovic, "FOXO transcription factors: key regulators of cell fate," Biochemical Society Transactions, vol. 34, part 5, pp. 722-726, 2006.

[28] H. Kurzen, I. Moll, R. Moll et al., "Compositionally different desmosomes in the various compartments of the human hair follicle," Differentiation, vol. 63, no. 5, pp. 295-304, 1998.

[29] H. Wu, J. R. Stanley, and G. Cotsarelis, "Desmoglein isotype expression in the hair follicle and its cysts correlates with type of keratinization and degree of differentiation," Journal of Investigative Dermatology, vol. 120, no. 6, pp. 1052-1057, 2003.

[30] H. Zali, "Proteomic analysis of gene expression in basal cell carcinoma," Iranian Journal of Dermatology, vol. 13, no. 4, pp. 112-117, 2010.
[31] A. S. Goldman, "The immune system of human milk: antimicrobial, antiinflammatory and immunomodulating properties," Pediatric Infectious Disease Journal, vol. 12, no. 8, pp. 664-672, 1993.

[32] P. Vural, M. Canbaz, and D. Selçuki, "Plasma antioxidant defense in actinic keratosis and basal cell carcinoma," Journal of the European Academy of Dermatology and Venereology, vol. 13, no. 2, pp. 96-101, 1999.

[33] M. A. El-Khalawany and A. A. Abou-Bakr, "Role of cyclooxygenase-2, ezrin and matrix metalloproteinase-9 as predictive markers for recurrence of basal cell carcinoma," Journal of Cancer Research and Therapeutics, vol. 9, no. 4, pp. 613-617, 2013.

[34] V. Bartos, D. Pokorny, O. Zacharova et al., "Recurrent basal cell carcinoma: a clinicopathological study and evaluation of histomorphological findings in primary and recurrent lesions," Acta Dermatovenerologica Alpina, Pannonica et Adriatica, vol. 20, no. 2, pp. 67-75, 2011.

[35] F. S. Bøgelund, P. A. Philipsen, and R. Gniadecki, "Factors affecting the recurrence rate of basal cell carcinoma," Acta Dermato-Venereologica, vol. 87, no. 4, pp. 330-334, 2007.

[36] H. I. Friedman, T. Williams, S. Zamora, and Z. A. Al-Assaad, "Recurrent basal cell carcinoma in margin-positive tumors," Annals of Plastic Surgery, vol. 38, no. 3, pp. 232-235, 1997.

[37] B. Zagrodnik, W. Kempf, B. Seifert et al., "Superficial radiotherapy for patients with basal cell carcinoma: recurrence rates, histologic subtypes, and expression of p53 and Bcl-2," Cancer, vol. 98, no. 12, pp. 2708-2714, 2003.

[38] A. Telliez, C. Furman, N. Pommery, and J.-P. Hénichart, "Mechanisms leading to COX-2 expression and COX-2 induced tumorigenesis: Topical therapeutic strategies targeting COX2 expression and activity," Anti-Cancer Agents in Medicinal Chemistry, vol. 6, no. 3, pp. 187-208, 2006.

[39] M. I. Patel, K. Subbaramaiah, B. Du et al., "Celecoxib inhibits prostate cancer growth: evidence of a cyclooxygenase-2independent mechanism," Clinical Cancer Research, vol. 11, no. 5, pp. 1999-2007, 2005.

[40] S. You, R. Li, D. Park et al., "Disruption of STAT3 by niclosamide reverses radioresistance of human lung cancer," Molecular Cancer Therapeutics, vol. 13, no. 3, pp. 606-616, 2014.

[41] J.-W. Tjiu, Y.-H. Liao, S.-J. Lin et al., "Cyclooxygenase-2 overexpression in human basal cell carcinoma cell line increases antiapoptosis, angiogenesis, and tumorigenesis," Journal of Investigative Dermatology, vol. 126, no. 5, pp. 1143-1151, 2006.

[42] H.-R. Park, S. K. Min, K. Min et al., "Differential expression of ezrin in epithelial skin tumors: cytoplasmic ezrin immunoreactivity in squamous cell carcinoma," International Journal of Dermatology, vol. 49, no. 1, pp. 48-52, 2010.

[43] Y.-C. Wei, C.-F. Li, S.-C. Yu et al., "Ezrin overexpression in gastrointestinal stromal tumors: an independent adverse prognosticator associated with the non-gastric location," Modern Pathology, vol. 22, no. 10, pp. 1351-1360, 2009.

[44] M. Bagheri, M. Eghtedari, M. Bagheri, B. Geramizadeh, and M. Talebnejad, "Expression of maspin and ezrin proteins in periocular Basal cell carcinoma," Dermatology Research and Practice, vol. 2014, Article ID 596564, 6 pages, 2014.

[45] S. Ilmonen, A. Vaheri, S. Asko-Seljavaara, and O. Carpen, "Ezrin in primary cutaneous melanoma," Modern Pathology, vol. 18, no. 4, pp. 503-510, 2005.

[46] A. G. Abdou, A. H. Maraee, E. M. M. El-Sayed, and N. F. Elnaidany, "Immunohistochemical expression of ezrin in 
cutaneous basal and squamous cell carcinomas," Annals of Diagnostic Pathology, vol. 15, no. 6, pp. 394-401, 2011.

[47] C. Delclaux, C. Delacourt, M.-P. D’Ortho, V. Boyer, C. Lafuma, and A. Harf, "Role of gelatinase B and elastase in human polymorphonuclear neutrophil migration across basement membrane," American Journal of Respiratory Cell and Molecular Biology, vol. 14, no. 3, pp. 288-295, 1996.

[48] B. Heissig, K. Hattori, S. Dias et al., "Recruitment of stem and progenitor cells from the bone marrow niche requires MMP-9 mediated release of Kit-ligand," Cell, vol. 109, no. 5, pp. 625-637, 2002.

[49] T. Kobayashi, H. Kim, X. Liu et al., "Matrix metalloproteinase9 activates TGF- $\beta$ and stimulates fibroblast contraction of collagen gels," American Journal of Physiology-Lung Cellular and Molecular Physiology, vol. 306, no. 11, pp. L1006-L1015, 2014.

[50] C. Pyke, E. Ralfkiær, P. Huhtala, T. Hurskainen, K. Danø, and K. Tryggvason, "Localization of messenger RNA for Mr 72,000 and 92,000 type IV collagenases in human skin cancers by in situ hybridization," Cancer Research, vol. 52, no. 5, pp. 1336-1341, 1992.

[51] W. G. Stetler-Stevenson, S. Aznavoorian, and L. A. Liotta, "Tumor cell interactions with the extracellular matrix during invasion and metastasis," Annual Review of Cell Biology, vol. 9, no. 1, pp. 541-573, 1993.

[52] V. Dumas, J. Kanitakis, S. Charvat, S. Euvrard, M. Faure, and A. Claudy, "Expression of basement membrane antigens and matrix metalloproteinases 2 and 9 in cutaneous basal and squamous cell carcinomas," Anticancer Research, vol. 19, no. 4, pp. 2929-2938, 1999.

[53] R. Glaser, R. Andridge, E. V. Yang et al., "Tumor site immune markers associated with risk for subsequent basal cell carcinomas," PLoS ONE, vol. 6, no. 9, Article ID e25160, 2011.

[54] D. A. Wong, G. A. Bishop, M. A. Lowes, B. Cooke, R. S. Barnetson, and G. M. Halliday, "Cytokine profiles in spontaneously regressing basal cell carcinomas," British Journal of Dermatology, vol. 143, no. 1, pp. 91-98, 2000.

[55] M. J. Hunt, G. M. Halliday, D. Weedon, B. E. Cooke, and R. S. Barnetson, "Regression in basal cell carcinoma: an immunohistochemical analysis," British Journal of Dermatology, vol. 130, no. 1, pp. 1-8, 1994.

[56] H. Ansarin, M. Daliri, and R. Soltani-Arabshahi, "Expression of p53 in aggressive and non-aggressive histologic variants of basal cell carcinoma," European Journal of Dermatology, vol. 16, no. 5, pp. 543-547, 2006.

[57] S. Bolshakov, C. M. Walker, S. S. Strom et al., "p53 mutations in human aggressive and nonaggressive basal and squamous cell carcinomas," Clinical Cancer Research, vol. 9, no. 1, pp. 228-234, 2003.

[58] M. Yu, D. Zloty, B. Cowan et al., "Superficial, nodular, and morpheiform basal-cell carcinomas exhibit distinct gene expression profiles," Journal of Investigative Dermatology, vol. 128, no. 7, pp. 1797-1805, 2008.

[59] B. G. Howell, N. Solish, C. Lu et al., "Microarray profiles of human basal cell carcinoma: insights into tumor growth and behavior," Journal of Dermatological Science, vol. 39, no. 1, pp. 39-51, 2005.

[60] J. Yang, S. A. Mani, J. L. Donaher et al., "Twist, a master regulator of morphogenesis, plays an essential role in tumor metastasis," Cell, vol. 117, no. 7, pp. 927-939, 2004.

[61] G. Kallergi, M. A. Papadaki, E. Politaki, D. Mavroudis, V. Georgoulias, and S. Agelaki, "Epithelial to mesenchymal transition markers expressed in circulating tumour cells of early and metastatic breast cancer patients," Breast Cancer Research, vol. 13, no. 3, article R59, 2011.

[62] M. Nakamura and Y. Tokura, "Epithelial-mesenchymal transition in the skin," Journal of Dermatological Science, vol. 61, no. 1, pp. 7-13, 2011.

[63] Y. Majima, S. Hirakawa, Y. Kito et al., "Twistl as a possible biomarker for metastatic basal cell carcinoma," Acta DermatoVenereologica, vol. 92, no. 6, pp. 621-622, 2012.

[64] E. Rosivatz, I. Becker, K. Specht et al., "Differential expression of the epithelial-mesenchymal transition regulators snail, SIP1, and twist in gastric cancer," The American Journal of Pathology, vol. 161, no. 5, pp. 1881-1891, 2002.

[65] A. Pizarro, "E-cadherin expression is frequently reduced in infiltrative basal cell carcinoma," The Journal of Dermatology, vol. 27, no. 12, pp. 804-805, 2000.

[66] T. V. Karelina, G. I. Goldberg, and A. Z. Eisen, "Matrilysin (PUMP) correlates with dermal invasion during appendageal development and cutaneous neoplasia," Journal of Investigative Dermatology, vol. 103, no. 4, pp. 482-487, 1994.

[67] K. Sasaki, S. Natsugoe, S. Ishigami et al., "Significance of Twist expression and its association with E-cadherin in esophageal squamous cell carcinoma," Journal of Experimental \& Clinical Cancer Research, vol. 28, article 158, 2009.

[68] K. Kashima, N. Ohike, S. Mukai, M. Sato, M. Takahashi, and T. Morohoshi, "Expression of the tumor suppressor gene maspin and its significance in intraductal papillary mucinous neoplasms of the pancreas," Hepatobiliary and Pancreatic Diseases International, vol. 7, no. 1, pp. 86-90, 2008.

[69] J. S. Reis-Filho, B. Torio, A. Albergaria, and F. C. Schmitt, "Maspin expression in normal skin and usual cutaneous carcinomas," Virchows Archiv, vol. 441, no. 6, pp. 551-558, 2002.

[70] A. G. Abdou, A. H. Maraee, M. A. E.-M. Shoeib, and A. M. A. Saida, "Maspin expression in epithelial skin tumours: an immunohistochemical study," Journal of Cutaneous and Aesthetic Surgery, vol. 4, no. 2, pp. 111-117, 2011.

[71] G. Zoccali, R. Pajand, P. Papa, G. Orsini, N. Lomartire, and M. Giuliani, "Giant basal cell carcinoma of the skin: literature review and personal experience," Journal of the European Academy of Dermatology and Venereology, vol. 26, no. 8, pp. 942-952, 2012.

[72] K. M. Olde Loohuis, A. Doeksen, and B. C. Vrouenraets, "Basal cell carcinoma: natural course if neglected," European Journal of Dermatology, vol. 21, no. 3, pp. 419-421, 2011.

[73] H. Tsukamoto, K. Hayashibe, Y. Mishima, and M. Ichihashi, "The altered expression of $\alpha$-smooth muscle actin in basal cell epithelioma and its surrounding stroma: with special reference to proliferating cell nuclear antigen expression and adenoid differentiation," British Journal of Dermatology, vol. 130, no. 2, pp. 189-194, 1994.

[74] P. A. Adegboyega, S. Rodriguez, and J. McLarty, "Stromal expression of actin is a marker of aggressiveness in basal cell carcinoma," Human Pathology, vol. 41, no. 8, pp. 1128-1137, 2010.

[75] M. M. Christian, R. L. Moy, R. F. Wagner, and A. Yen-Moore, "A correlation of alpha-smooth muscle actin and invasion in micronodular basal cell carcinoma," Dermatologic Surgery, vol. 27, no. 5, pp. 441-445, 2001.

[76] S.-I. Motegi, K. Yamada, and O. Ishikawa, "Twistl in tumor cells and $\alpha$-smooth muscle actin in stromal cells are possible biomarkers for metastatic giant basal cell carcinoma," The Journal of Dermatology, vol. 40, no. 8, pp. 661-663, 2013. 
[77] S. T. Oh, H. S. Kim, N. J. Yoo, W. S. Lee, B. K. Cho, and J. Reichrath, "Increased immunoreactivity of membrane type-1 matrix metalloproteinase (MT1-MMP) and $\beta$-catenin in highrisk basal cell carcinoma," British Journal of Dermatology, vol. 165, no. 6, pp. 1197-1204, 2011.

[78] M. El-Bahrawy, N. El-Masry, M. Alison, R. Poulsom, and M. Fallowfield, "Expression of $\beta$-catenin in basal cell carcinoma," British Journal of Dermatology, vol. 148, no. 5, pp. 964-970, 2003.

[79] D. Ron and P. Walter, "Signal integration in the endoplasmic reticulum unfolded protein response," Nature Reviews Molecular Cell Biology, vol. 8, no. 7, pp. 519-529, 2007.

[80] D. M. Ferrari, P. Van Nguyen, H. D. Kratzin, and H.-D. Söling, "ERp28, a human endoplasmic-reticulum-lumineal protein, is a member of the protein disulfide isomerase family but lacks a CXXC thioredoxin-box motif," European Journal of Biochemistry, vol. 255, no. 3, pp. 570-579, 1998.

[81] C. Cheretis, F. Dietrich, I. Chatzistamou et al., "Expression of ERp29, an endoplasmic reticulum secretion factor in basal-cell carcinoma," American Journal of Dermatopathology, vol. 28, no. 5, pp. 410-412, 2006.

[82] I. F. Bambang, S. Xu, J. Zhou, M. Salto-Tellez, S. K. Sethi, and D. Zhang, "Overexpression of endoplasmic reticulum protein 29 regulates mesenchymal-epithelial transition and suppresses xenograft tumor growth of invasive breast cancer cells," Laboratory Investigation, vol. 89, no. 11, pp. 1229-1242, 2009.

[83] C. L. Chaffer, E. W. Thompson, and E. D. Williams, "Mesenchymal to epithelial transition in development and disease," Cells Tissues Organs, vol. 185, no. 1-3, pp. 7-19, 2007.

[84] G. Ponti, G. Bertazzoni, L. Pastorino et al., "Proteomic analysis of PTCH1+/- fibroblast lysate and conditioned culture media isolated from the skin of healthy subjects and nevoid basal cell carcinoma syndrome patients," BioMed Research International, vol. 2013, Article ID 794028, 8 pages, 2013.

[85] K. Yamamoto, H. Yoshihashi, N. Furuya et al., "Further delineation of 9q22 deletion syndrome associated with basal cell nevus (Gorlin) syndrome: report of two cases and review of the literature," Congenital Anomalies, vol. 49, no. 1, pp. 8-14, 2009.

[86] S. Abe, K. Kabashima, J.-I. Sakabe et al., "Coincident two mutations and one single nucleotide polymorphism of the PTCH1 gene in a family with naevoid basal cell carcinoma syndrome," Acta Dermato-Venereologica, vol. 88, no. 6, pp. 635636, 2008.

[87] A. Valin, S. Barnay-Verdier, T. Robert et al., " $P T C H 1^{+/-}$dermal fibroblasts isolated from healthy skin of Gorlin syndrome patients exhibit features of carcinoma associated fibroblasts," PLoS ONE, vol. 4, no. 3, p. e4818, 2009.

[88] G. Ponti, G. Bertazzoni, L. Pastorino et al., "Proteomic analysis of PTCH1+/- fibroblast lysate and conditioned culture media isolated from the skin of healthy subjects and nevoid basal cell carcinoma syndrome patients," BioMed Research International, vol. 2013, Article ID 794028, 8 pages, 2013.

[89] M. B. Weiss, E. V. Abel, M. M. Mayberry, K. J. Basile, A. C. Berger, and A. E. Aplin, "TWIST1 is an ERK1/2 effector that promotes invasion and regulates MMP-1 expression in human melanoma cells," Cancer Research, vol. 72, no. 24, pp. 6382-6392, 2012. 


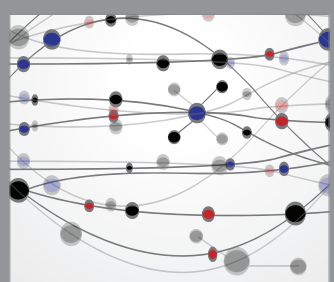

The Scientific World Journal
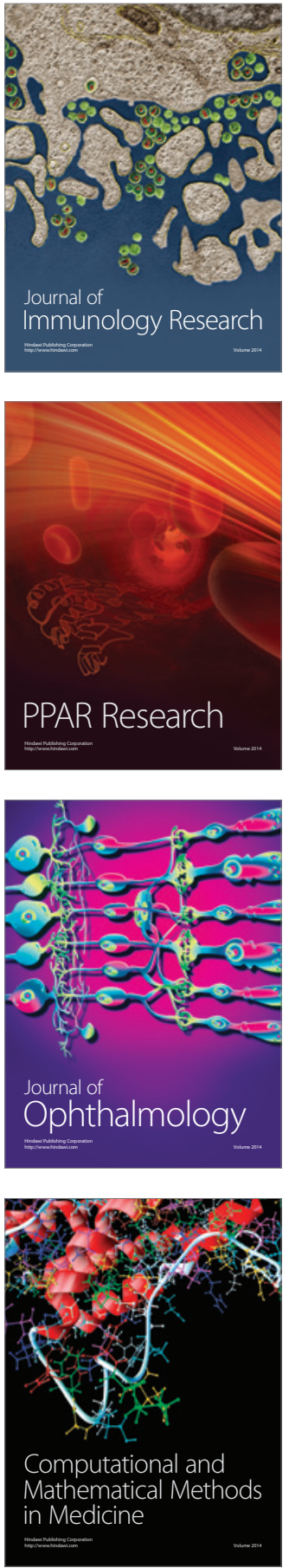

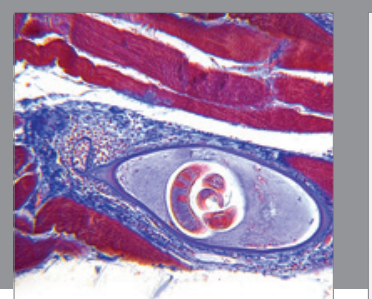

Gastroenterology Research and Practice

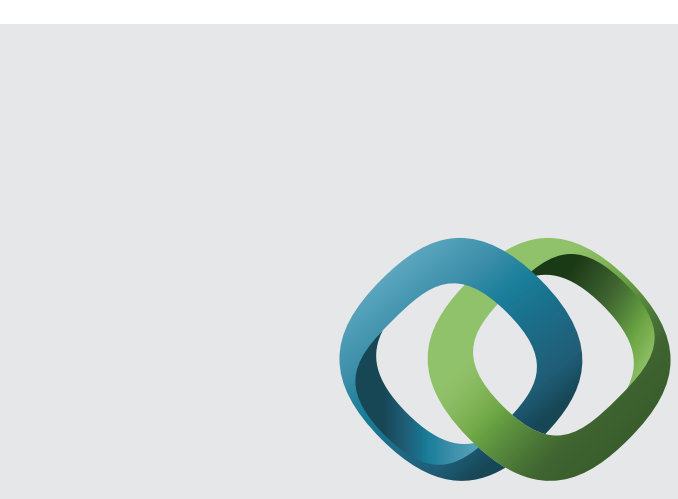

\section{Hindawi}

Submit your manuscripts at

http://www.hindawi.com
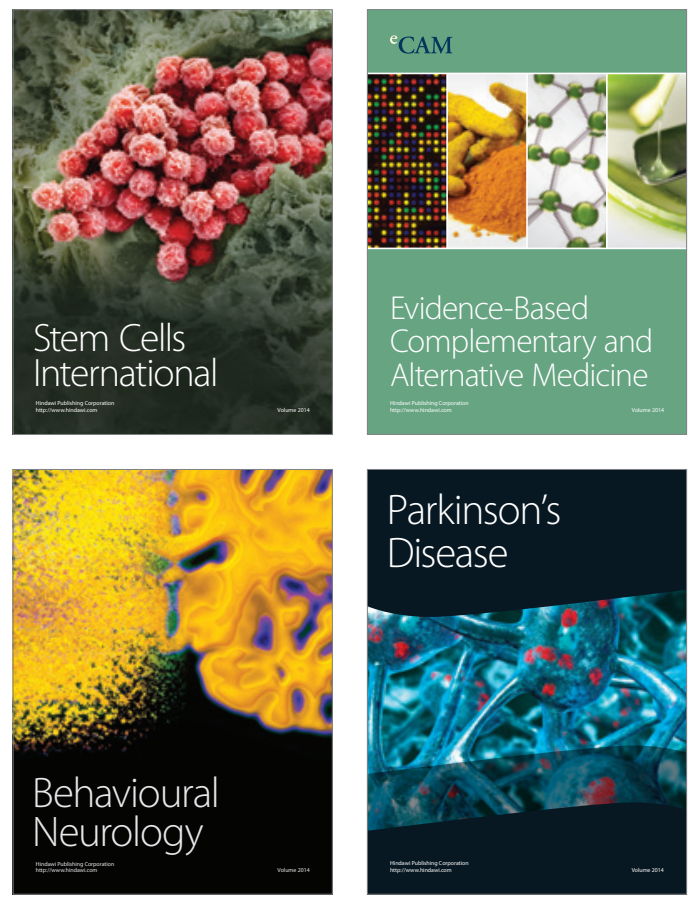
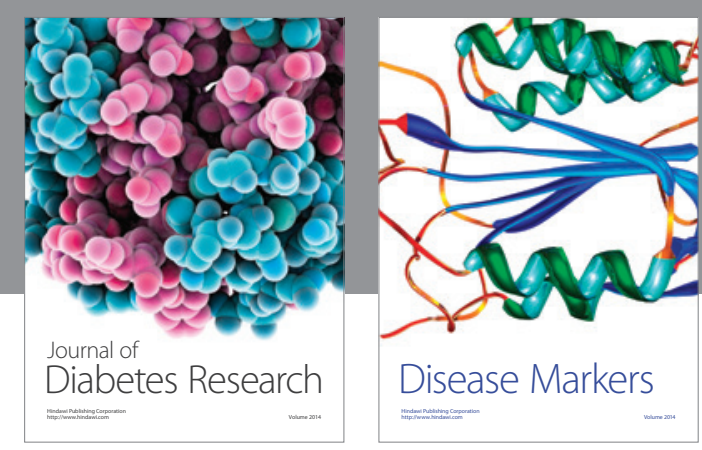

Disease Markers
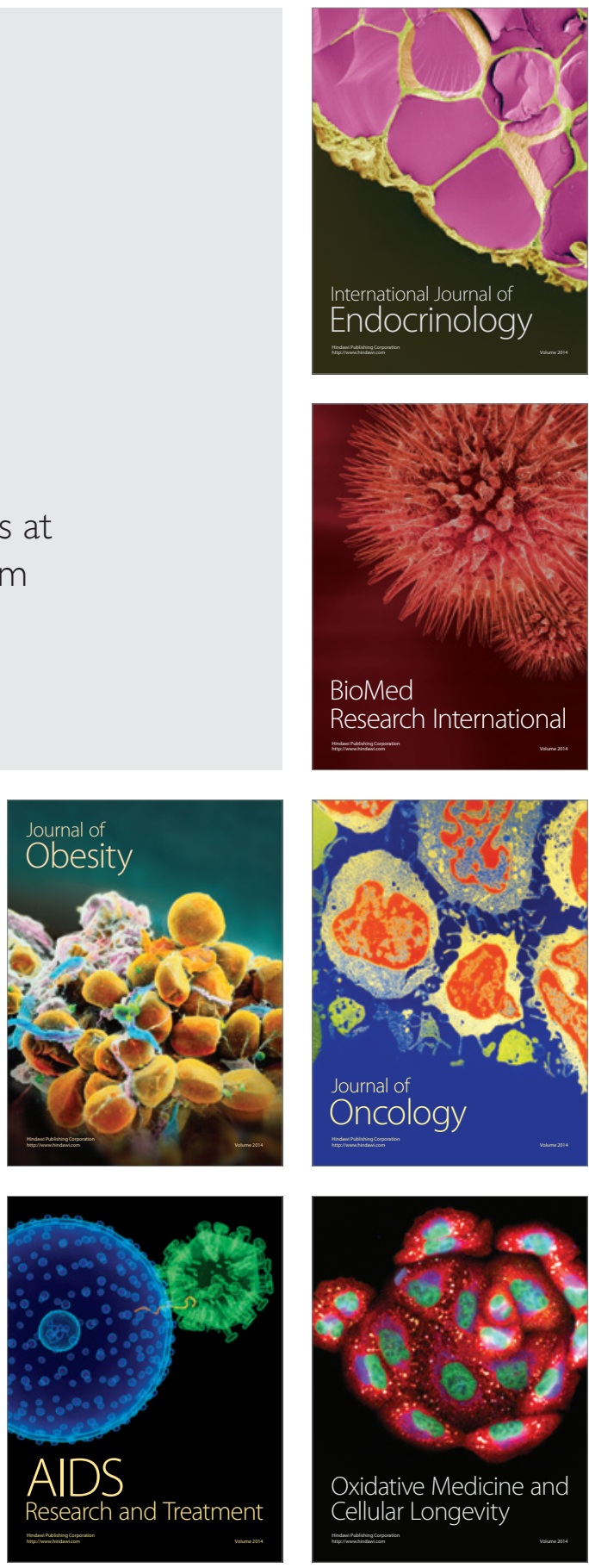